Cahiers « Mondes anciens »

ANCIENS

Histoire et anthropologie des mondes anciens

11 | 2018

La « civilisation » : critiques épistémologique et historique

\title{
Comment Marcel Mauss croyait à l'origine romaine de la Civilisation
}

How Marcel Mauss believed in the roman origin of Civilization

\section{Florence Dupont}

\section{(2) OpenEdition}

Journals

Édition électronique

URL : http://journals.openedition.org/mondesanciens/2175

DOI : 10.4000/mondesanciens. 2175

ISSN : 2107-0199

Éditeur

UMR 8210 Anthropologie et Histoire des Mondes Antiques

Référence électronique

Florence Dupont, « Comment Marcel Mauss croyait à l'origine romaine de la Civilisation », Cahiers «Mondes anciens » [En ligne], 11 | 2018, mis en ligne le 19 mars 2018, consulté le 20 avril 2019. URL: http://journals.openedition.org/mondesanciens/2175; DOI : 10.4000/mondesanciens.2175

Ce document a été généré automatiquement le 20 avril 2019

\section{(c) $($ ) $(9)$}

Les Cahiers «Mondes Anciens » sont mis à disposition selon les termes de la licence Creative Commons Attribution - Pas d'Utilisation Commerciale - Pas de Modification 4.0 International. 


\section{Comment Marcel Mauss croyait à l'origine romaine de la Civilisation}

How Marcel Mauss believed in the roman origin of Civilization

Florence Dupont

\section{Le dernier texte de Mauss, Une catégorie de l'esprit humain : la notion de personne, ou l'invention de la personne à Rome}

1 Le terme latin persona est souvent placé à l'origine de la notion moderne et occidentale de personne comme si l'étymologie imposait cette préhistoire ${ }^{1}$. La plus connue de ces reconstructions est le texte de Marcel Mauss Une catégorie de l'esprit humain: la notion de personne, celle de "moi" ", article originalement publié en 1938 dans Journal of the Royal Anthropological Institute, vol. $68^{2}$.

2 L'essai de Mauss prétend décrire la généalogie occidentale de la conscience individuelle, d'un Moi personnel et unique, irréductible à un statut ou à l'appartenance à un groupe, signifié par le terme français "personne». Forme supérieure de civilisation, cette invention aurait été initiée par Rome et se serait accomplie dans la modernité occidentale. Mauss écrit à l'époque du personnalisme de la revue Esprit d'Emmanuel Mounier qui donne à la notion de personne une force philosophique et politique, sousjacente à son essai. Il s'agit pour ce mouvement de résister à la montée des totalitarismes, niant la personne au profit de la race ou de la classe, mais aussi de se démarquer de l'individualisme bourgeois ${ }^{3}$. Le devoir des intellectuels est de rétablir la personne, c'est-àdire l'homme en tant qu'acteur libre et responsable. Les menaces sur la personne sont une menace sur la « civilisation».

3 La personne de Mauss est une construction sociale : il dit dans son essai qu'il « est un sujet d'histoire sociale» (p. 6). Cependant Mauss, en substituant la personne à l'individu dans le grand récit de la civilisation, ne change pas de paradigme. Comme Durkheim, il 
s'inscrit dans une pensée évolutionniste : l'individu est pour Durkheim le marqueur de la civilisation, c'est-à-dire de la modernité occidentale, pour Mauss, c'est la personne.

4 L'objet de cet article n'est pas de critiquer la pensée de Mauss à l'œuvre dans cet essai, cette critique a été faite brillamment et sévèrement par d'autres ${ }^{4}$. Certes cet essai de Mauss est la publication d'une conférence destinée à un large public, qui ne pouvait s'embarrasser d'un apparat académique rebutant. Dans le contexte politique mondial, ce texte avait en outre une dimension militante. Il n'est pas question d'ignorer ces deux circonstances ayant présidé à son écriture et de reprocher à Mauss de ne pas avoir écrit un essai scientifiquement rigoureux. Cela dit, il faudrait éviter d'en faire un texte fondateur de la sociologie et de le confronter aux autres écrits de Mauss. Son intérêt est ailleurs.

5 Précisément, en effet, en " lâchant prise ", en se laissant aller à des parcours intuitifs et rapides, Mauss révèle un impensé commun aux intellectuels français de son temps : la civilisation européenne est le modèle de toute civilisation en ce qui concerne les droits de la personne et à l'origine de ce miracle civilisationnel, il y a Rome, nécessairement. Une des catégories emblématiques de notre civilisation, «la personne», viendrait en droite ligne et par la force de l'étymologie, d'une catégorie romaine, persona. Le terme latin de persona recélerait déjà en partie l'essence de cette catégorie appelée à être progressivement révélée au cours du grand récit de la personne.

6 Or tout antiquisant lisant les quelques pages consacrées à l'étape romaine de la personne ne peut être qu'étonné de la désinvolture de Mauss à l'égard des documents historiques et ethnographiques, même dans le contexte évoqué précédemment. Nulle part ailleurs ce grand savant ne fait preuve d'une telle légèreté. C'est pour nous, historiens et anthropologues de Rome, une énigme que cet article va tenter sinon de résoudre, du moins de problématiser, en resituant l'étape romaine de la persona, dans sa stratégie narrative $e^{5}$. Nous verrons que Rome est la clef de voûte de sa démonstration et que ce qui nous semble aujourd'hui un manque évident de rigueur, s'explique par la nécessité que tout commence à Rome.

\section{À Rome se rencontrent l'horizontalité ethnologique et la verticalité historique}

7 L'essai de Mauss débute par un préambule ethnologique qui cherche une préhistoire de la personne dans des sociétés primitives où la catégorie s'esquisserait sans avoir encore de nom. Les sociétés prises en compte sont juxtaposées et comparées dans une intemporalité horizontale. Mauss reprend quelques études réalisées par d'autres que lui sur des sociétés amérindiennes et australiennes, afin d'établir un catalogue de formes du moi et à partir de là construire le paradigme d'une pré-personne, qui serait propre aux sociétés sans histoire.

Il fait l'hypothèse qu'à l'origine de notre notion de personne, il y aurait un masque, sans doute déjà parce que persona en latin signifiait «masque». Prisonnier volontaire de l'étymologie de la langue française, il va voir du côté des sociétés primitives qui toutes, dit-il, pratiquent des rituels masqués. Il rencontre des masques dans des cultes d'ancêtres, généralise cette configuration en associant un nom propre au masque et conclut que les porteurs de masque intériorisent ce qui leur est transmis avec leur nom : des statuts et des rôles, des représentations et des figures. Ce premier paradigme est celui 
du « personnage ». Le " personnage » réunit un masque rituel, un ancêtre clanique et des privilèges sociaux accordés à celui qui le porte comme descendant de cet ancêtre. Le masque permet à cet homme de se distinguer par son rôle social du reste de son clan et de tous les autres nobles. Ainsi, "tout un immense ensemble de sociétés est arrivé à la notion de personnage, de rôle rempli par l'individu dans des drames sacrés comme il joue un rôle dans la vie familiale. La fonction a déjà créé la formule depuis des sociétés très primitives, jusqu'à nos sociétés à nous » (p. 15).

fait l'enquête est faussée par un présupposé général, le latin est la langue pour dire le personnage d'avant la personne et ce qui le concerne. Ainsi Mauss pour parler de la marque personnelle que les Kwakliutl ${ }^{6}$ Amérindiens mettent sur leurs objets écrit : « Les plats, les fourchettes, les cuivres, tout est blasonné, animé, fait partie de la persona du propriétaire et de la familia, des res de son clan » (p.13). La persona désigne tout d'un coup le personnage, la pré-personne. La notion avait précédé le mot et l'attendait.

10 La seconde partie de son essai est verticale, il passe d'une série de descriptions à un grand récit, l'histoire occidentale de la personne qui commence avec Rome. Le trajet doit mener du masque rituel d'un ancêtre, modèle élaboré dans le champ ethnographique, persona, au sujet psychologique, unique et conscient de l'être, la personne. Le Moi va se constituer au cours de l'histoire de la civilisation européenne, depuis Rome jusqu'à nos jours avec, après Rome, les étapes obligées de la philosophie grecque, du christianisme etc. Chaque période historique fait progresser la notion de persona jusqu'à ce qu'elle devienne une personne.

11 Rome est donc le moment crucial de l'essai, car Rome aurait fait le pas décisif qui engageait la civilisation occidentale, c'est-à-dire la Civilisation, dans le long chemin menant à la reconnaissance de la personne, individu singulier, pourvu d'une conscience de soi et d'une histoire propre. Elle aurait fait passer la persona du masque concret à une notion juridique abstraite, persona, articulant le temps arrêté de l'ethnographie au temps actif de l'histoire. Le terme persona appartiendrait comme Rome à ces deux temporalités. La Rome archaïque d'abord décrite en termes ethnologiques donne naissance à la Rome classique, décrite en termes historiques.

La Civilisation vs les civilisations primitives est caractérisée par ce passage de l'ethnographie à l'histoire, car entrer dans le temps historique c'est être capable d'évoluer et d'inventer, non seulement techniquement mais aussi moralement ce qui serait le propre de la civilisation occidentale et qui en fait la civilisation des civilisations ${ }^{7}$.

Certes d'autres civilisations, selon Mauss, ont accédé au temps historique, mais elles se sont arrêtées en route, comme l'Inde et la Chine, qui ne sont pas allées jusqu'à élaborer une notion complète du Moi. Elles se seraient figées à un moment de leur histoire. Les spécialistes de ces deux espaces culturels réagissent fermement à cette partie de l'essai ${ }^{8}$ en dénonçant la méconnaissance des faits historiques et une simplification outrancière de sociétés complexes et diversifiées à partir de quelques écrits philosophiques ou religieux. Le sinologue parle "d'erreurs substantielles ". L'indianologue reproche à Mauss son évolutionnisme. Les termes qui lui servent à rendre compte du Moi en Inde sont entachés de présupposés monothéistes; Mauss ne conçoit, en effet, qu'une seule forme de conscience de soi et toute altérité est projetée sur un axe chronologique dont la fin est l'Europe du xx siècle. Autrement dit, Mauss ne pense pas dans cet essai en anthropologue de la diversité des cultures. Ces deux critiques adressées à Mauss sont aussi valables pour son chapitre sur Rome. Il y fait de « substantielles erreurs », ces erreurs étant dues moins 
à l'état des savoirs historiques à son époque qu'à la place qu'il attribue a priori à Rome dans la civilisation occidentale et qui dévoie sa démonstration.

Rome est donc le lieu et le moment où l'horizontal s'articule au vertical, où le récit de Mauss passe de l'ethnographie à l'histoire. Articulation particulièrement laborieuse et peu crédible, qui trahit, plus que tout le reste, l'acte de foi qui préside à cet essai : la civilisation occidentale a fait entrer le Monde dans le progrès moral et le respect de la personne humaine par l'invention du sujet conscient de son identité unique.

La stratégie de Mauss impose que les Romains aient d'abord été des primitifs comme les autres et qu'ils aient possédé des masques rituels, puis qu'ils soient devenus civilisés pour inventer la personne juridique d'où provient la personne psychologique actuelle. Le paradigme ethnologique élaboré précédemment ne peut s'appliquer qu'à une Rome archaïque, préhistorique et perdue. Il a été constitué dans ce but. Du sauvage au civilisé, le fil conducteur est le mot persona dont le sens premier, primitif, serait le masque et le sens second, civilisé, la personne juridique.

L'argumentation était difficile, faute de documents; c'est pourquoi les faits attestés relèvent souvent de la rumeur académique, "vous savez tous que », le récit s'appuie sur des affirmations de la seule autorité de Mauss «je pense que », «il me semble que » et nous verrons plus loin que les citations sont même parfois tronquées.

\section{Mauss confond persona et imago sous le français « masque»}

Pour affirmer que la persona des Romains était à la fois un masque de théâtre et un masque d'ancêtre, Mauss fait appel à la rumeur savante, en connivence avec son auditoire :

Vous savez tous combien est normale, classique la notion de persona latine : masque, masque tragique, masque rituel et masque d'ancêtre. Elle est donnée au début de la civilisation Latine.

Or tout latiniste sait bien qu'il y a au moins deux types de masques à Rome : la persona qui est un masque de théâtre et l'imago qui est un masque d'ancêtre, sans qu'ils n'aient rien de commun entre eux. L'imago est l'empreinte à la cire des traits du mort, reproduisant le visage dans sa singularité, la persona est un rôle, le masque indique que l'acteur joue un roi, une reine de tragédie ou un vieillard revêche, un esclave malin, une courtisane de comédie.

On remarquera la façon dont Mauss commence par une catégorie générale, ethnocentrique, «le masque », intraduisible en latin, qu'il détaille ensuite en différentes sortes de masques, de telle sorte que tous semblent appartenir à la même catégorie posée initialement qui du coup se met à exister. Pour faire cependant le lien entre les deux, sans toutefois jamais parler ici d'imago, il reprend l'hypothèse d'une persona venant du grec prosopon, à travers l'étrusque en s'appuyant sur Benveniste et Meillet (p. 18-19). Puis il ajoute, pratiquant l'amalgame, que les archéologues ont retrouvé de nombreux masques funèbres étrusques ${ }^{10}$.

Les mystérieux Étrusques hantent toute l'histoire de la Rome archaïque; dès qu'il y a un chaînon manquant entre Rome et la Grèce, ils servent à combler l'absence. C'est ainsi qu'entre prosopon et persona, il y aurait un démon infernal, dénommé Phersu, apparenté par son nom à Perséphone, la souveraine des morts, et à Persée, détenteurs l'un et l'autre 
de la tête de la Gorgone. Cette hypothèse s'appuie sur des fresques étrusques retrouvées dans trois tombes, toutes trois de la seconde moitié du vie siècle av. J.-C., localisées à Tarquinia: la tombe des Augures, la tombe du Polichinelle, et la tombe des Jeux Olympiques. On voit sur chacune un individu cagoulé, avec deux fois l'inscription Phersu. Certains ont voulu croire que le mot phersu désignait le masque du personnage. Ce qui est improbable. Généralement, en effet, le nom placé à côté d'un personnage peint est un nom propre, identifiant ce personnage, parfois reconnaissable aussi grâce à un accessoire; comme Héraklès et sa massue peuvent être associés à l'inscription Herkle (Héraclès en étrusque). Donc ce personnage, caractérisé par cette cagoule ou masque très visibles, doit s'appeler Phersu. Que ce nom propre soit devenu un nom commun désignant ce qui était le propre de Phersu, par une dérivation en - na, fréquente en étrusque, rien ne s'y oppose, mais rien non plus le prouve. En tout cas cela ne permet pas de démontrer que persona désignait un masque funéraire utilisé dans des rituels romains, alors que les masques funéraires sont toujours appelés imagines.

21 Ayant fait de persona un masque "primitif ", Mauss cherche vainement un rituel romain qui utiliserait une persona d'ancêtre. Il est étonnant qu'il ait raté le funus indictivum où les masques des ancêtres portés par des acteurs défilent en procession dans Rome. Il va dans les environs de Rome et rencontre un rituel falisque ${ }^{11}$, celui des Hirpi Sorani $i^{12}$ qui chaque année marchaient sur des charbons ardents au mont Soracte, en l'honneur d'Apollon Soranus, ou peut-être du dieu infernal Dis pater. La rhétorique de Mauss est retorse, il écrit (p. 19) :

D'abord, on trouve chez eux des traces définies d'institutions du genre des cérémonies des clans, des masques, des peintures dont les acteurs s'ornent suivant les noms qu'ils portent [ce qui est pure invention de Mauss]. Au moins l'un des grands rituels de la Rome très ancienne [ce n'est pas un grand rituel, et il n'est pas très ancien, car il date seulement de l'époque où les Falisques ont reçu la citoyenneté romaine, après les guerres sociales, au $\mathrm{II}^{\mathrm{e}} \mathrm{s}$. av. J.-C.] correspond exactement au type commun dont nous avons décrit les formes accusées. C'est celui des Hirpi Sorani, des loups du Soracte (Hirpi = nom du loup en Samnite). Les gens des familles qui portaient ce titre marchaient sur des charbons ardents au sanctuaire de la déesse Feronia, et jouissaient de privilèges et d'exemption d'impôts. Sir James G. Frazer a déjà supposé [la référence à une supposition de Frazer, même avec son titre de noblesse, n'est pas très sérieuse] que c'est le reste d'un ancien clan, devenu confrérie, portant noms, peaux, masques.

Sa méthode est la même que précédemment: poser une catégorie générale, ethnographiquement pré-construite, ici l'existence à Rome de rituels claniques avec masques et peintures, l'illustrer par un exemple qui semble une preuve. Mauss ajoute ce dont il a besoin pour sa démonstration. Hirpus voulait certes dire « loup » en falisque ou en sabellique, langues en fait éloignées du samnite qui est un dialecte osque. Cette erreur, qui est de peu d'importance, vient elle-même de Servius, commentateur de Virgile, qui avait confondu les Hirpini, tribu samnite avec les Hirpi Sorani. Quant à Féronia, d'où sortelle? En tout cas il n'est pas question dans ce rituel de masque ou de déguisements. Les Hirpi Sorani ne sont pas exemptés seulement de charges mais aussi de service militaire, ce qui les marginalise dans la vie publique. Cette exception est une exclusion et non un privilège.

23 À partir de là, l'exposé devient du roman. Mauss suit le loup à la trace et dramatise sa découverte (p. 19) :

Mais il y a plus; il semble bien que nous sommes ici en présence du mythe même de Rome. Acca Larentia, la vieille, la mère des Lares, fêtée aux Larentalia (décembre), n'est 
autre que l'indigitamentum, le nom secret de la Louve Romaine, mère de Romulus et

de Remus (Ov., Fastes, 1, 55 sq.) communs à tous. "De là à la notion de personne, il n'y a qu'un pas.», écrit-il. La métaphore est allègre, la démonstration qui suit est laborieuse. Les droits et privilèges des chefs de clan auraient donc été étendus à tous, sous le nom de droit civil. Mauss manipule une persona qui n'a jamais existé et invente une histoire fondée sur des «je conçois », « je crois » et des raccourcis historiques (p. 19) :

Je conçois que les légendes comme celle du consul Brutus et de ses fils, de la fin du droit du pater de tuer ses fils, ses sui, traduisent l'acquisition de la persona par les fils, du vivant même de leur père. L'important est le moment où la persona abandonne le support matériel du masque pour devenir une catégorie abstraite. Je crois que la révolte de la plèbe, le plein droit de cité qu'acquérirent [sic] - après les fils des familles sénatoriales - tous les membres plébéiens des gentes, fut décisive. Furent citoyens Romains tous les hommes libres de Rome, tous eurent la persona civile.

La dernière phrase n'a aucun sens. Veut-il dire que les citoyens romains avaient accès au jus ciuile? Ou aux mêmes droits politiques? Mauss semble ignorer les différents types de citoyenneté et confond l'accès au droit civil, droit privé, avec une ciuitas qui est très variable et peut comporter des droits politiques restreints, comme la ciuitas sine suffragio.

Certes le nom persona appartient bien à la langue juridique romaine mais il n'est pas une catégorie englobant tous les êtres humains libres, pourvus de droit, par opposition aux esclaves, car les esclaves sont parfois des personae:

Et quidem summa divisio de iure personarum haec est, quod omnes homines aut liberi sunt aut servi.

Globalement le droit des personnes se divisent en deux : car tous les hommes sont ou libres ou esclaves ${ }^{13}$.

À Rome le droit reconnaît différentes catégories de personae, il s'agit de tous les êtres humains, libres ou non, femmes et enfants, opposés aux animaux. Par ailleurs les animaux susceptibles d'être possédés sont dans la même catégorie que les esclaves, res mancipi. La persona ne désigne donc pas une catégorie à laquelle des droits seraient attachés. La 
persona est sans contenu a priori, elle est une catégorie classificatoire intervenant selon la situation sur laquelle le droit doit agir, elle ne relève pas d'une ontologie.

Or Mauss essentialise la persona et lie le droit à la "personne », alors qu'à Rome le droit est activé selon les circonstances ${ }^{14}$. Il écrit (p. 20-21):

Le caractère personnel du droit était fondé, et persona était devenu synonyme de la vraie nature de l'individu. Le droit à la persona est fondé. Seul en est exclu l'esclave. Servus personam non habet. Il n'a pas de personnalité. Il n'a pas son corps, il n'a pas d'ancêtres, de nom, de cognomen, de biens propres.

31 Cette phrase marque la fin de l'analyse de la persona romaine juridique et ouvre, après une étape stoïcienne, le passage au christianisme. C'est lui qui donnera, selon Mauss, une persona à l'esclave, qui jusqu'ici n'en avait pas. Or cette affirmation repose sur une citation tronquée de Justinien ${ }^{15}$ " servus personam non habet » qui sonne comme une vérité générale définissant la nature de l'esclave. La citation complète est :

Cum standi in judicio servus personam non habeat, procuratorem ad lites non habet, licet ad negotia habeat.

L'esclave ne pouvant pas se présenter dans un tribunal, il ne peut pas être représenté par un procurateur dans un procès, mais il peut avoir un procurateur pour faire des affaires.

Cette phrase n'a pas le sens que lui donne Mauss. Standi définit persona dans l'espace du tribunal : l'esclave ne peut pas comparaître comme plaignant ou comme défenseur (stare in judicio), et ne peut pas non plus avoir un procurateur au tribunal, même s'il peut faire des affaires (negotia) ; un procurateur, dans ce cas-là, agira alors pour lui, comme pour un fils de famille qui n'a pas été émancipé, et ses droits seront alors en affaires les mêmes que ceux d'un homme libre. Ce qui rend difficile pour un esclave d'agir en droit n'est pas sa nature, mais la question des cautions, car son maitre est responsable financièrement de tout ce qu'il fait. Le droit romain, on le voit, utilise une fiction afin de contourner la difficulté. En affaires (negotia) l'esclave est comme un fils de famille non émancipé.

La persona juridique est donc un rôle dans une action du droit, et non un ensemble de droits liés à la personne. Dans d'autres passages, il est question de la persona servi, fonction d'esclave. Ainsi, au théâtre comme en droit, la persona désigne un rôle joué dans une actio. C'est aussi ce sens que Cicéron donne à persona dans une phrase célèbre qui oppose le rôle de l'acteur et celui de l'orateur ${ }^{16}$ :

Qua re nolite existimare me ipsum, qui non heroum ueteres casus fictosque luctus uelim imitari atque adumbrare dicendo neque actor sim alienae personae, sed auctor meae, cum mihi M. Aquilius in ciuitate retinendus esset, quae in illa causa peroranda fecerim, sine magno dolore fecisse.

C'est pourquoi croyez bien que moi même je ne prétends pas, quand je plaide, représenter et imiter les malheurs des héros antiques et leurs deuils imaginaires, je ne cherche pas à jouer le rôle d'un autre, mais ce que je veux, c'est être l'auteur de mon rôle. Quand je devais conserver Manlius Aquilius à notre cité et que j'ai prononcé la péroraison, ma douleur était grande.

Agir en droit, jouer une pièce (agere fabulam), plaider dans un procès (agere causam) : celui qui agit prend un rôle, une persona temporaire. La persona ne s'inscrit pas dans l'être, elle n'est pas liée à la personne. Étant un rôle, chacun peut le jouer, il suffit que le préteur légitime la fiction. 


\section{Un bilan globalement négatif}

\section{BIBLIOGRAPHIE}

Ando C. (2013), L'Empire et le Droit. Invention juridique et réalités historiques à Rome, Paris.

Carrithers M., Collins St. et Lukes St. éd. (1985), The Category of the Person: Anthropology, Philosophy, History, Cambridge.

David J.-M. (2003), « Le prix de la voix : remarques sur la clause d'exclusion des praecones de la table d'Héraclée », Hantos, Theodora. Laurea internationalis : Festschrift für Jochen Bleicken zum 75. Geburtstag, p. 81-106.

Faure-Ribreau M. (2011), « L'identité en question. Étude du terme persona dans l'œuvre de Cicéron », BAGB 1-2, p. 126-169.

Guérin Ch. (2009 et 2011), Persona. L'élaboration d'une notion rhétorique au I ${ }^{\text {er }}$ siècle av. J.-C., vol. I : Antécédents grecs et première rhétorique latine; vol. II : Théorisation cicéronienne de la persona oratoire, Paris.

Housset E. (2007), La vocation de la personne. L'histoire du concept de personne de sa naissance augustinienne à sa redécouverte phénoménologique, Paris. 
Ildefonse F. (2009), « Être une personne. La personne en Grèce ancienne », Terrain 52, p. 64-77.

Jannot J. R. (1993), «Phersu, Phersuna, Persona. À propos du masque étrusque », Spectacles sportifs et scéniques dans le monde étrusco-italique. Actes de la table ronde de Rome (3-4 mai 1991), Rome, p. 281-320.

Mauss M. (1938), « Une catégorie de l'esprit humain : la notion de personne, celle de "moi" ", Journal of the Royal Anthropological Institute 68, p. 263-281.

Meyerson I. dir. (1973), Problèmes de la personne, Colloque du Centre de recherches de psychologie comparative, EPHE VI ${ }^{e}$ Section, Paris-La Haye.

Mounier E. (2000), Écrits sur le personnalisme, Paris.

Nédoncelle M. (1948), « Prosôpon et persona dans l'Antiquité classique. Essai de bilan linguistique », RSR 22, p. 277-299.

Pahud S. (2013), Le statut de l'esclave et sa capacité à agir dans le domaine contractuel. Etude de droit romain de l'époque classique [en ligne], thèse de doctorat de l'université de Lausanne, GenèveZurich-Bâle. URL : https://serval.unil.ch/notice/serval:BIB_675169FD2C8B

Rissanen M (2012) « The Hirpi Sorani and the wolf cults of Central Italy », Arctos 46, p. 115-135.

Rougemont D. de (1934), Politique de la personne, Paris.

Tarot C. (2008), « Problématiques maussiennes de la personne », Cahiers internationaux de sociologie 124, p. 21-39.

\section{NOTES}

1. Par exemple : Nédoncelle 1948 ; Meyerson 1973 ; Housset 2007 ; Ildefonse 2009.

2. Repris dans Sociologie et Anthropologie, Paris, 1970, p. 333-362. Le texte est accessible en ligne à l'adresse : http://classiques.uqac.ca/classiques/mauss_marcel/ mauss_marcel.html ; Nous nous référerons à la pagination de la publication en ligne.

3. Mounier 2000 ; Rougemont 1934.

4. Pour une analyse critique de cet essai voir Carrithers et al. 1985. Je remercie Alessandro Buccheri qui m'a communiqué cette référence bibliographique, essentielle et à laquelle je me reporterai souvent.

5. Dans Carrithers et al. 1985, Michael Carrithers affirme que la force persuasive du texte de Mauss tient à sa stratégie (p. 234).

6. Les Kwakliutl sont un peuple amérindien de Colombie-Britannique (Canada).

7. C'est « le grand partage » selon la désormais célèbre formule de Michel de Certeau.

8. Dans Carrithers et al. 1985, voir Mark Elvin, chap. 8 et Alexis Sanderson, chap. 9.

9. Dans Carrithers et al. 1985, voir Mark Elvin, «Between the earth and the heaven. Conception of the self in China », p. 156 et suiv.

10. Jannot 1993. Étude archéologiquement très documentée mais l'analyse des documents est contestable, c'est pourquoi nous ne suivons pas ses conclusions sur Phersu.

11. Au Nord est du Latium.

12. Sur les Hirpi Sorrani voir Rissanen 2012 ; Pline l'ancien, Histoire naturelle, 7, 19: Haud procul urbe Roma in Faliscorum agro familiae sunt paucae quae vocantur Hirpi. Hae sacrificio 
annuo, quod fit ad montem Soractem Apollini, super ambustam ligni struem ambulantes non aduruntur et ob id perpetuo senatus consulto militiae omniumque aliorum munerum vacationem habent. Sur le territoire des Falisques, non loin de Rome, il y a quelques familles qui portent le nom de Hirpi. Ces familles, lors d'un sacrifice annuel à Apollon, au pied du mont Soracte, marchent, sans se brûler sur un lit de charbons ardents. Un senatus consulte les dispense pour toujours du service militaire et toute autre charge.

13. Gaius, Institutions, I, 9-12.

14. Voir David 2003 et Ando 2013.

15. Justinien, Apodectes, II, XVIII. Pahud 2013. Cette citation interprétée à tord comme une définition de l'esclave est reprise à l'infini.

16. Cicéron, De oratore, II, 194. Voir Guérin 2009 et 2011 ; Faure-Ribreau 2011.

\section{RÉSUMÉS}

Marcel Mauss dans son fameux essai Une catégorie de l'esprit humain : la notion de personne, celle de «moi» fait de la personne une catégorie témoin de l'évolution de la civilisation occidentale. « Personne » ayant pour étymologie persona, il place Rome à l'articulation d'une première analyse ethnographique de la notion de personne avec une analyse historique, linéaire, limitée à l'Occident. C'est à Rome que le masque primitif, ébauche d'une première singularité attachée à celui qui en hérite et le porte, serait devenu une catégorie du droit. Or cette reconstitution est historiquement non fondée et le chapitre consacré à la persona romaine développe une intuition de Mauss sans véritable argumentation. Comment un tel savant a-t-il pu ainsi perdre toute rigueur ? L'hypothèse suggérée est que la place assignée à Rome (et à la Grèce) dans le grand récit de la civilisation occidentale parasite les études historiques et ethnographiques : il «faut » que Rome (ou la Grèce) soit à l'origine des valeurs de cette civilisation.

In his famous essay, Une catégorie de l'esprit humain: la notion de personne, celle de "moi", Marcel Mauss transforms the "person" into a witness of the evolution of Western civilization. With the word "person", whose etymology comes from persona, Mauss firmly places Rome at the hinge of an ethnographic analysis of the notion of "person", coupled with a linear historical analysis limited to the West. It is in Rome that the primitive mask, which uniquely singles out he who inherits and wears it, would have become a legal category. However, this reconstitution is historically unfounded and, in the chapter dedicated to the Roman "persona", Mauss elaborates based solely on an intuition and one he fails to substantiate. How could such a fine scholar manage to lose all intellectual integrity? My hypothesis is that the place assigned to Rome (and Greece, for that matter) in the great story of Western civilization poisons historical and ethnographical studies; it is imperative that Rome (or Greece) be at the origins of this civilization's values.

\section{INDEX}

Keywords : Marcel Mauss, person, Rome, mask, civilization, evolutionism

Mots-clés : Marcel Mauss, personne, Rome, masque, civilisation, évolutionnisme 


\section{AUTEUR}

\section{FLORENCE DUPONT}

Université Paris Diderot-Paris 7 - latinjussieu@aol.com 